\section{Themenkomplex I: Definitionen, Epidemiologie und natürlicher Verlauf}

W. Schepp, H.-D. Allescher, T. Frieling, M. Katschinski, P. Malfertheiner, C. Pehl, U. Peitz, W. Rösch, J. Hotz †

\section{Definitionen}

\section{Nosologische Definition Konsens}

Eine allgemein gültige und verbindliche nosologische Definition der gastroösophagealen Refluxkrankheit gibt es bisher nicht. In Anlehnung an den internationalen Konsens - The Genval Workshop Report 1999 [1] - wird die folgende Definition empfohlen:

Eine gastroösophageale Refluxkrankheit liegt vor, wenn ein Risiko für organische Komplikationen durch einen gesteigerten gastroösophagealen Reflux und/oder eine signifikante Störung des gesundheitsbezogenen Wohlbefindens (Lebensqualität) infolge der Refluxbeschwerden besteht (C).

\section{Kommentar}

Organische Komplikationen einer Refluxkrankheit können sich im Bereich der Speiseröhre (z.B. Ösophagitis, Stenosen, Barrett) sowie extraösophageal (siehe Leitlinien „Extraösophageale Manifestationen der gastroösophagealen Refluxkrankheit“) manifestieren. Eine Refluxkrankheit wird als wahrscheinlich angesehen, wenn Refluxsymptome (siehe Leitlinien „Diagnostik der Refluxkrankheit“) mindestens $1 \times /$ Woche [2] bis $2 \times /$ Woche [1] auftreten und mit einer Beeinträchtigung der Lebensqualität einhergehen. Allerdings können auch Refluxbeschwerden, die seltener auftreten, zu einer relevanten Minderung der Lebensqualität führen [3].

\section{Gastroösophageale Refluxkrankheit Konsens}

Die gastroösophageale Refluxkrankheit der Speiseröhre wird im medizinischen Sprachgebrauch als GERD abgekürzt. Unter dem Begriff werden die verschiedenen Manifestationen nicht erosive Refluxkrankheit (NERD), erosive Ösophagitis verschiedener Schweregrade (ERD), Barrett-Ösophagus und extraösophageale Manifestationen subsumiert (C).

\section{Kommentar}

Unter pathophysiologischen und therapeutischen Aspekten wird zwischen primärer und sekundärer (siehe Abschnitt sekundäre Refluxkrankheit auf der nächsten Seite) Refluxkrankheit unterschieden.

\section{Nichterosive gastroösophageale Refluxkrankheit (NERD) Konsens}

GERD ohne endoskopisch nachweisbare Läsionen wird als nichterosive gastroösophageale Refluxkrankheit definiert (NERD). NERD liegt nur vor, wenn Beschwerden die Lebensqualität beeinträchtigen. NERD kann die Lebensqualität ( $\mathrm{Q} O \mathrm{~L})$ ähnlich stark beeinträchtigen wie ERD (B).

\section{Kommentar}

Die Unterscheidung zwischen NERD und ERD ist weder durch das Ausmaß der Beschwerden oder der Beeinträchtigung der Lebensqualität möglich noch durch das Ansprechen auf PPI-Therapie oder durch nachweisbar andere Empfindlichkeit gegen Säureexposition [1, 4-6]. Bei einem Teil der Patienten liegt eine Überlappung mit der funktionellen Dyspepsie bzw. dem Reizdarmsyndrom, gelegentlich mit psychopathologischen Bedingungen, vor [5]. Die NERD kann auch bei Patienten mit refluxbedingten laryngopulmonalen Manifestationen (Laryngitis, chronischer Bronchitis, Asthma bronchiale) bestehen [5].

\section{Epidemiologie}

\section{Prävalenz und Inzidenz der GERD Konsens}

Die Prävalenz der GERD mit allen Manifestationsformen liegt in den westlichen Industrieländern bei 10-20\%. Die epidemiologischen Daten sprechen für eine Zunahme der GERD-Inzidenz in den letzten Jahrzehnten für alle Manifestationsformen, wenngleich prospektive Daten fehlen (B).

\section{Kommentar}

In epidemiologischen Studien schwanken die Prävalenzangaben für mindestens einmal pro Woche auftretendes Sodbrennen zwischen 11 und $18 \%$ [2, 7-9]. Auswertungen einer amerikanischen Diagnosestatistik sprechen für eine Häufigkeitszunahme der ERD in den letzten Jahrzehnten [10]. Auch eine steigende Inzidenz der NERD ist aufgrund allgemeiner Erfahrungen und Retrospektivanalysen sehr wahrscheinlich [11], wenngleich prospektive kontrollierte Daten fehlen. Ebenso wird für extraösophageale Manifestationen trotz fehlender prospektiver kontrollierter Studien ein absoluter Häufigkeitsanstieg angenommen [2, 12]. Eine familiäre Häufung für die verschiedenen Formen der GERD ist wahrscheinlich [13]. Für eine vermutete Abnahme der Inzidenz von Strikturen in den letzten 2 Dekaden, möglicherweise infolge besserer medikamentöser Therapie, sind die Literaturdaten uneinheitlich [14-17].

\section{Geschlechts- und Altersverteilung der GERD Konsens}

Die Daten aus populationsbasierten Studien sprechen für eine vergleichbare Prävalenz von Refluxbeschwerden bei Frauen und Männern (B).

Die Prävalenz der GERD ist nicht altersspezifisch, ein Altersgipfel der Erstmanifestation ist in der Literatur nicht eindeutig belegt (B).

\section{Kommentar}

Epidemiologische Studien mit Befragungen großer Bevölkerungsstichproben zeigen ein identisches Geschlechtsverhältnis oder wechselnd ein Vorwiegen des weiblichen oder männlichen Geschlechts [2, 7, 18-22]. Klinikbasierte Studien lassen vermuten, dass Männer häufiger eine ERD aufweisen [10, 14, 23, 24]. Beim Barrett-Ösophagus überwiegt das männliche Geschlecht mit ca. 60 - 70\% [14, 25]. Extraösophageale Manifestationen sind bei beiden Geschlechtern gleich häufig [2]. 
Es ist offen, ob Patienten mit höhergradiger ERD bzw. BarrettÖsophagus im Mittel ein höheres Alter aufweisen als Patienten mit NERD bzw. leichtgradiger ERD [2, 10, 14, 23, 24, 26]. Ebenso ist umstritten, ob die Prävalenz von Sodbrennen mit dem Alter zunimmt $[2,7,20,22,23]$.

\section{Sozioökonomie}

\section{Lebenserwartung \\ Konsens}

Die unkomplizierte GERD hat keinen Einfluss auf die Lebenserwartung (B).

\section{Kommentar}

Selbst eine ERD Grad II-III hat nur geringen Einfluss auf das Überleben [27]. Auch beim Barrett-Ösophagus ist wegen des seltenen Adenokarzinoms eine generell erniedrigte Lebenserwartung nicht nachgewiesen [28]. Lebensbedrohliche Komplikationen der GERD (Blutung, Strikturen) treten zwar nur bei einem kleinen Prozentsatz der GERD-Patienten auf [2], wegen der hohen Prävalenz der GERD ist aber von durchaus beträchtlichen Absolutzahlen auszugehen. So stieg in England und Wales zwischen 1968 und 1991 die Mortalität infolge benigner Ösophaguserkrankungen an [29].

\section{Kostenaufwand}

\section{Konsens}

Aufgrund des vergleichbar hohen Leidensdruckes von NERD und ERD sind ähnliche ärztliche und medikamentöse Leistungen zur Wiederherstellung der Lebensqualität erforderlich (C).

\section{Kommentar}

Für eine adäquate Behandlung von NERD und ERD werden vergleichbare PPI-Dosen benötigt [5]. Die direkten jährlichen Fallkosten für ärztliche Leistungen und Medikamente werden mit 185 CHF bis 510 \$ angegeben [30, 31]. Daraus errechnen sich für 7-10 Mio. behandlungsbedürftige GERD-Patienten in Deutschland Aufwendungen von ca. 3-4 Milliarden Euro pro Jahr. Eine verlässliche prospektive Kostenanalyse fehlt.

\section{Arbeitsunfähigkeit, Berentung}

\section{Konsens}

Bei Patienten mit NERD/geringgradiger ERD spielen indirekte Kosten für Arbeitsunfähigkeit bzw. Berentung eine erhebliche Rolle und rechtfertigen die Kosten einer angemessenen medikamentösen Therapie (B).

\section{Kommentar}

Mit einer beschwerdebedingten Beeinträchtigung ihrer Arbeitsfähigkeit ist bei 23 bis 33\% der Refluxpatienten zu rechnen [32, 33]. Die indirekten Kosten für GERD durch Arbeitsunfähigkeit und gelegentliche Berentung sind ähnlich hoch wie die direkten Therapiekosten [32, 34, 35]. Im Vergleich zu ambulanten Ulkuspatienten vor der H.-p.-Eradikationsära sind die Kosten für GERD vergleichbar bzw. sogar höher zu veranschlagen [36].

\section{Natürlicher Verlauf}

\section{Keine wesentliche Progredienz Konsens}

Das Stadium der NERD/ERD ist bei > 95\% der Patienten über viele Jahre nicht progredient, regelmäßige endoskopische Verlaufskontrollen sind daher nicht erforderlich. Eine spontane langfristige Rückbildung der GERD ist selten (B).

\section{Kommentar}

Prävalenz und Schwere von Refluxsymptomen sind unabhängig von der Dauer der Beschwerden und vom Alter der Patienten. Bei den meisten Patienten mit ERD und NERD bleibt das Stadium der Erkrankung bei Diagnosestellung in den folgenden Jahrzehnten stabil [1, 2, 4, 37-40]. Endoskopische Verlaufskontrollen nach Absetzen der Therapie sind daher verzichtbar, symptomatische Rezidive weisen meist denselben endoskopischen Schweregrad auf wie die Index-Gastroskopie bei Erstdiagnose [39]. Andererseits sind Spontanremissionen v. a. bei höhergradiger ERD selten [41, 42], Rezidive nach Absetzen einer Therapie dagegen sehr häufig $[43,44]$.

\section{Sekundäre Refluxkrankheit Konsens}

Spezifische pathophysiologische Störungen aufgrund anderer Krankheitsbilder können zur GERD führen und werden als sekundäre Refluxkrankheit bezeichnet (C).

\section{Kommentar}

Als Ursachen sind akzeptiert: Magenausgangsstenose und funktionelle Gastroparese, Gravidität [45], Magenverweilsonde [46], Zollinger-Ellison-Syndrom [47], Sjögren/Sicca-Syndrom [48], Sklerodermie [49], neuromuskuläre Erkrankungen sowie geistige Behinderungen (Übersicht bei [50]).

\section{Assoziation mit anderen Erkrankungen Konsens}

Über eine Assoziation von GERD mit anderen gastrointestinalen und/oder extragastrointestinalen Erkrankungen wird in der Literatur berichtet, ohne dass direkte kausale pathogenetische Mechanismen gesichert sind (C).

\section{Kommentar}

Dies gilt z.B. für die distale Gastrektomie [51], die Cholezystektomie [52], die Peritonealdialyse [53], Zöliakie [54], Diabetes mellitus [55, 56], Koronarinsuffizienz [57], M. Parkinson [58], psychiatrische Erkrankungen [59] und Schlafapnoe [60-62].

\section{Medikamente \\ Konsens}

Medikamente können die Symptomatik der GERD verstärken, da sie die komplexe Funktion des gastroösophagealen Verschlussmechanismus und der ösophagealen Clearance stören können (C).

\section{Kommentar}

$\mathrm{Zu}$ diesen Medikamenten gehören Kalziumantagonisten, Nitropräparate, Theophylline (Verstärkung eines refluxbedingten Asthmas!), Anticholinergika, Psychopharmaka, orale Kontrazeptiva und pfefferminzölhaltige Präparate u.a. [63]. Kalziumanta- 
gonisten, Nitropräparate und Anticholinergika können zudem durch Reduktion der ösophagealen Kontraktionsamplituden die ösophageale Säure-Clearance beeinträchtigen [64]. Darüber hinaus reduzieren Anticholinergika die Sekretion Bikarbonat enthaltenden Speichels und vermindern dadurch die Säureneutralisation im Ösophagus [65]. Refluxpatienten scheinen zudem ein erhöhtes Strikturrisiko zu tragen bei Einnahme von ASS oder NIARD [66].

\section{Literatur}

${ }^{1}$ Dent J, Brun J, Frederick A et al. An evidence-based appraisal of reflux disease management - the Genval Workshop Report. Gut 1999; 44 (Suppl 2): S1 - 16

2 Locke GR, Talley NJ, Fett SL et al. Prevalence and clinical spectrum of gastroesophageal reflux: a population-based study in Olmsted county, Minnesota. Gastroenterology 1997; 112: $1448-1456$

${ }^{3}$ Dimenas E, Carlsson G, Glise H et al. Relevance of norm values as part of the documentation of quality of life instruments for use in upper gastrointestinal disease. Scand J Gastroenterol 1996; 31 (Suppl 221): $8-13$

${ }^{4}$ McDougall NI, Johnston BT, Kee F et al. Natural history of reflux oesophagitis: a 10 year follow-up of its effect on patient symptomatology and quality of life. Gut 1996; 38: $481-486$

${ }^{5}$ Quigley EM. Non erosive reflux disease: part of the spectrum of gastro-oesophageal reflux disease, a component of functional dyspepsia, or both? Eur J Gastroenterol Hepatol 2001; 13 (Suppl 1): S13 - 18

${ }^{6}$ Kulig M, Leodolter A, Vieth M et al. Quality of life in relation to symptoms in patients with gastro-oesophageal reflux disease - an analysis based on the ProGERD initiative. Aliment Pharmacol Ther 2003; 18: $767-776$

${ }^{7}$ Isolauri J, Laippala P. Prevalence of symptoms suggestive of gastrooesophageal reflux disease in an adult population. Ann Med 1995; 27: $67-70$

${ }^{8}$ Raiha I, Impivaara O, Seppala M et al. Determinants of symptoms suggestive of gastroesophageal reflux disease in the elderly. Scand J Gastroenterol 1993; 28: 1011 - 1014

${ }^{9}$ Louis E, DeLooze D, Deprez P et al. Heartburn in Belgium: prevalence, impact on daily life, and utilization of medical resources. Eur J Gastroenterol Hepatol 2002; 14: 279-284

10 Sonnenberg A, Massey BT, Jacobson SJ. Hospital discharges resulting from esophagitis among Medicare beneficiaries. Dig Dis Sci 1994; 39: $183-188$

${ }^{11}$ Bardhan KD, Royston C, Nayyar AK. Reflux rising! A disease in evolution? Gastroenterology 2000; 118: A 478

12 El-Serag HB, Sonnenberg A. Extraesophageal complications of gastroesophageal reflux disease in US veterans. Gastroenterology 1997; 113: $755-760$

${ }^{13}$ Trudgill NJ, Kapur KC, Riley SA. Familial clustering of reflux symptoms. Am J Gastroenterology 1999; 94: 1172 - 1178

${ }^{14}$ Lööf L, Gotell P, Elfberg B. The incidence of reflux oesophagitis. A study of endoscopy reports from a defined catchment area in Sweden. Scand J Gastroenterol 1993; 28: 113 - 118

15 Agnew SR, Pandya SP, Reynolds RPE et al. Predictors for frequent esophageal dilations of benign peptic strictures. Dig Dis Sci 1996; 5: 931-936

${ }^{16}$ Jornod P, Dorta G, Pantoflickova D et al. Peptic esophageal stricture: a vanishing complication of reflux esophagitis? Gastroenterology 2000; 118: A 484

${ }^{17}$ Nayyar AK, Roysten C, Bardhan KD. Oesophageal acid-peptic strictures in the histamine $\mathrm{H} 2$ receptor antagonist and proton pump inhibitor era. Dig Liver Dis 2003; 35: $143-150$

${ }^{18}$ Kennedy T, Jones R. The prevalence of gastrooesophageal reflux symptoms in a UK population and the consultation behaviour of patients with these symptoms. Aliment Pharmacol Ther 2000; 14: 1589-1594

${ }^{19}$ Ruth M, Mansson I, Sandberg N. The prevalence of symptoms suggestive of esophageal disorders. Scand J Gastroenterol 1991; 26: 73-81
${ }^{20}$ Stanghellini V. Three-month prevalence rates of gastrointestinal symptoms and the influence of demographic factors: Results from the Domestic/International Gastroenterology Surveillance Study (DIGEST). Scand J Gastroenterol 1999; 231 (Suppl): 20-28

${ }^{21}$ Kay L, Jorgensen T, Jensen KH. Epidemiology of abdominal symptoms in a random population: prevalence, incidence, and natural history. Eur J Epidemiol 1994; 10: 559-566

${ }^{22}$ Bolin TD, Korman MG, Hansky J et al. Heartburn: community perceptions. J Gastroenterol Hepatol 2000; 15: 35 - 39

${ }^{23}$ Voutilainen M, Sipponen P, Mecklin JP et al. Gastroesophageal reflux disease: prevalence, clinical, endoscopic and histopathological findings in 1.128 consecutive patients referred for endoscopy due to dyspeptic and reflux symptoms. Digestion 2000; 61: 6-13

${ }^{24}$ Carlsson R, Dent J, Watts R et al. Gastro-oesophageal reflux disease in primary care: an international study of different treatment strategies with omeprazole. Eur J Gastroenterol Hepatol 1998; 10: 119-124

${ }^{25}$ Spechler SJ. Epidemiology and natural history of gastroesophageal reflux disease. Digestion 1992; 51 (suppl 1): 24-29

${ }^{26}$ Triadafilopoulos G, Sharma R. Features of symptomatic gastroesophageal reflux disease in elderly patients. Am J Gastroenterol 1997; 92: 2007-2011

${ }^{27}$ Rantanen TK, Salo JA. Gastroesophageal reflux disease as a cause of death: analysis of fatal cases under conservative treatment. Scand J Gastroenterol 1999; 34: 229-233

28 Van der Burgh A, Dees J, Hop WCJ et al. Oesophageal cancer is an uncommon cause of death in patients with Barrett's oesophagus. Gut 1996; 39: 5-8

${ }^{29}$ Panos MZ, Walt RP, Stevenson C et al. Rising death rate from non-malignant disease of the oesophagus (NMOD) in England and Wales. Gut 1995; 36: $488-491$

${ }^{30}$ Schwenkglenks M, Marbet UA, Szucs TD. Epidemiology and costs of gastroesophageal reflux disease in Swizerland: a population-based study. Soz Präventivmed 2004; 49: 51 -61

${ }^{31}$ Bloom BS, Jayadevappa R, Wahl P et al. Time trends in cost of caring for people with gastroesophageal reflux disease. Am J Gastroenterol 2001; 96 (suppl): S64-69

32 Wahlquist P. Symptoms of gastroesophageal reflux disease, perceived productivity, and health-related quality of life. Am J Gastroenterol 2001; 96 (Suppl): S57-61

${ }^{33}$ Dean BB, Crawley JA, Schmitt CM et al. The burden of illness of gastrooesophageal reflux disease: impact on work productivity. Aliment Pharmacol Ther 2003; 17: 1309-1317

${ }^{34}$ Henke CJ, Levin TR, Henning JM et al. Work loss costs due to peptic ulcer disease and gastroesophageal reflux disease in a health maintenance organization. Am J Gastroenterol 2000; 95: 787-792

${ }^{35}$ Glise H. Quality of life and cost of therapy in reflux disease. Scand J Gastroenterol 1995; 30 (suppl 210): 38-42

${ }^{36}$ Levin TR, Schmittdiel JA, Kunz K et al. Costs of acid-related disorders to a health maintenance organization. Am J Med 1997; 103: 520-528

37 Pace F, Santalucia F, Bianchi Porro G. Natural history of gastro-oesophageal reflux disease without oesophagitis. Gut 1991; 32: $845-848$

38 Aste H, Bonelli L, Ferraris R et al. Gastroesophageal reflux disease. Relationship between clinical and histological features. Dig Dis Sci 1999; 44: $2412-2418$

${ }^{39}$ Kuster E, Ros E, Toledo-Pimentel V et al. Predictive factors of the longterm outcome in gastro-oesophageal reflux disease: six-year followup of 107 patients. Gut 1994; 35: 8-14

${ }^{40}$ Olloyo JB, Monnier P, Fontolliet C et al. The natural history, prevalence and incidence of reflux esophagitis. Gullet 1993; 3: 3-10

${ }^{41}$ Isolauri J, Luostarinen M, Isolauri E et al. Natural course of gastroesophageal reflux disease: 17-22 year follow-up of 60 patients. Am J Gastroenterol 1997; 92: 37-41

${ }^{42}$ Schindlbeck NE, Klauser AG, Berghammer G et al. Three year follow up of patients with gastrooesophageal reflux disease. Gut 1992; 33: $1016-1019$

${ }^{43}$ Robinson N, Lanza F, Avner D et al. Effective maintenance treatment of reflux esophagitis with low-dose lansoprazole: a randomized, doubleblind, placebo controlled trial. Ann Intern Med 1996; 124: 859- 867

${ }^{44}$ Vigneri S, Termini R, Leandro G et al. A comparison of five maintenance therapies for reflux esophagitis. N Engl J Med 1995; 333: $1106-1110$

45 Richter JE. Gastroesophageal reflux disease during pregnancy. Gastroenterol Clin North Am 2003; 32: 235-261 
${ }^{46}$ Wilmer A, Tack J, Frans E et al. Duodenogastroesophageal reflux and esophageal mucosal injury in mechanically ventilated patients. Gastroenterology 1999; 116: $1293-1299$

47 Miller LS, Vinayek R, Frucht H et al. Reflux esophagitis in patients with Zollinger-Ellison syndrome. Gastroenterology 1990; 98: 341 - 346

${ }^{48}$ Korsten MA, Rosman AS, Fishbein S et al. Chronic xerostomia increases esophageal acid exposure and is associated with esophageal injury. Am J Med 1991; 90: 701 - 706

${ }^{49}$ Shoenut JP, Wielers A, Micflikier AB. The extent and pattern of gastrooesophageal reflux in patients with scleroderma oesophagus: the effect of low-dose omeprazole. Aliment Pharmacol Ther 1993; 7: 509-513

${ }^{50}$ Böhmer CJM, Klinkenberg-Knol EC, de Niezen-Boer MC et al. Gastroesopheageal reflux disease in intellectually disabled individuals: how often, how serious, how manageable? Am J Gastroenterol 2000; 95: $1868-1872$

${ }^{51}$ Fujiwara Y, Nakagawa K, Kusunoki M. Gastroesophageal reflux after distal gastrectomy: possible significance of the angle of His. Am J Gastroenterol 1998; 93: $11-15$

52 Rothwell JF, Lawlor P, Byrne PJ. Cholecystectomy-induced gastroesophageal reflux: is it reduced by the laparoscopic approach ? Am J Gastroenterol 1997; 92: $1351-1354$

${ }^{53}$ Cekin AH, Boyacioglu S, Gursoy M et al. Gastroesophageal reflux disease in chronic renal failure patients with upper GI symptoms: multivariate analysis of pathogenetic factors. Am J Gastroenterol 2002; 97: $1352-1356$

${ }^{54}$ Iovino P, Ciacci C, Sabbatini F et al. Esophageal impairment in adult celiac disease with steatorrhea. Am J Gastroenterol 1998; 93: $1243-1249$
${ }^{55}$ Lluch I, Ascaso JF, Mora F et al. Gastroesophageal reflux in diabetes mellitus. Am J Gastroenterol 1999; 94: 919-924

${ }^{56}$ Murray FE, Lombard MG, Ashe J et al. Esophageal function in diabetes mellitus with special reference to acid studies and relationship to peripheral neuropathy. Am J Gastroenterol 1987; 82: 840-843

${ }^{57}$ Mehta AJ, de Caestecker JS, Camm AJ et al. Gastro-oesophageal reflux in patients with coronary artery disease: how common is it and does it matter? Eur J Gastroenterol Hepatol 1996; 8: 973 - 978

${ }^{58}$ Avidan B, Sonnenberg A, Giblovich $\mathrm{H}$ et al. Reflux symptoms are associated with psychiatric disease. Aliment Pharmacol Ther 2001; 15: $1907-1912$

${ }^{59}$ Ing AJ, Ngu MC, Breslin AB. Obstructive sleep apnea and gastroesophageal reflux. Am J Med 2000; 108 (Suppl 4a): 120S-125S

${ }^{60}$ Rasche K, Sanner B, Schäfer T et al. Sleep, breathing and gastroesophageal reflux. Dtsch Med Wochenschr 1997; 122: 659-663

61 Penzel T, Becker HF, Brandenburg U et al. Arousal in patients with gastro-oesophageal reflux and sleep apnea. Eur Respir J 1999; 14: $1266-1270$

62 Lagergren J, Bergstrom R, Adami Ho et al. Association between medications that relax the lower esophageal sphincter and risk for esophageal adenocarcinoma. Ann Intern Med 2000; 133: 165-175

63 Achem SR, Kolts BE. Current medical therapy for esophageal motility disorders. Am J Med 1992; 92 (suppl 5A): 98S-105S

${ }^{64}$ Helm JF, Dodds WJ, Hogan WJ et al. Acid neutralizing capacity of human saliva. Gastroenterology 1982; 83: 69-74

${ }^{65}$ Kim SL, Hunter JG, Wo JM et al. NSAIDs, aspirin, and esophageal strictures: are over-the-counter medications harmful to the esophagus? J Clin Gastroenterol 1999; 29: 32 - 34 